\title{
On the (extra)Galactic origin(s) of the lceCube neutrino signal
}

\section{Julia Becker Tjus*}

Theoretische Physik IV, Fakultät für Physik \& Astronomie, Ruhr-Universität, Bochum Ruhr Astroparticle and Plasma Physics Center (RAPP Center)

E-mail: julia.tjus@rub.de

\begin{abstract}
It has been 3 years since a first evidence for the existence of high-energy astrophysical neutrinos was announced by the IceCube Collaboration (Aartsen et al, Science, Nov 2013). Since then, there has been significant progress concerning both the experimental advancement and the theoretical interpretation of these data. In this talk, Galactic and extragalactic scenarios are reviewed taking into account most recent data and multimessenger modeling of the sources. In particular, it will be motivated in this paper that (a) both Galactic sources and gamma-ray bursts (at least in their prompt emission phase) are too weak to make up the total signal; (b) Starburst galaxies and active galactic nuclei are candidates that meet the criteria; (c) tensions between neutrino and gamma-ray data indicate that a larger fraction of the detected high-energy neutrinos actually might come from high-density regions in which both cosmic rays and high-energy photons are absorbed.
\end{abstract}

Neutrino Oscillation Workshop

4 - 11 September, 2016

Otranto (Lecce, Italy)

\footnotetext{
* Speaker.
} 


\section{Introduction}

The identification of sources of high-energy neutrinos promises the possibility of a direct detection channel of the sources of cosmic rays: high-energy neutrinos are produced in cosmic ray interactions with matter or photon fields, $C R+N / C R+\gamma \rightarrow \pi / K / \ldots \rightarrow(\ldots) v$. High-energy neutrinos are detected by IceCube in the energy range of $\sim \mathrm{TeV}$ to $\sim \mathrm{PeV}$, which corresponds to a cosmic ray energy range of $20 \mathrm{TeV}$ to $20 \mathrm{PeV}$, assuming that about 1/20th of the entire cosmic ray energy is transferred to the neutrino, see e.g. [1] and references therein.

The detected neutrino spectrum can be approximated as [2]

$$
E_{v}^{2} \cdot \frac{d N}{d E_{v}} \approx 10^{-8} \mathrm{GeV}^{-1} \mathrm{~cm}^{-2} \mathrm{~s}^{-1} \mathrm{sr}^{-1}
$$

This number is consistent with the general picture that cosmic ray sources typically have optical depths $(\tau)$ for photohadronic or hadron-hadron interactions that are much less than one, $\tau \ll 1$. Recent data from the IceCube experiment allow for even more detailed conclusions, as discussed in the paragraphs below.

Signal strength While an evaluation of the combination of all analyses with an astrophysical signal within IceCube indicates a steep spectrum of $E^{-2.5}$ above $20 \mathrm{TeV}$ [3], a dedicated analysis of track-like muon-neutrino events results in a best-fit of $E^{-2.13}$ above $200 \mathrm{TeV}$ [4]. A spectral hardening of the flux between 20 and $200 \mathrm{TeV}$ neutrino energy could therefore be possible. This energy range corresponds to $\mathrm{PeV}$ cosmic ray energies. While these first indications need to be substantiated by further data. Such a spectral hardening would indicate the presence of two population.

Isotropy The current data set of four years IceCube data contains 54 events. These show no significant clustering. In particular, there is no evident clustering in the Galactic plane. The large fraction of events outside of the Galactic Plane indicates that there needs to be a component that is not focussed in the plane, so either sources in the Galactic halo or extragalactic sources. As an example, 7 out of the 13 detected muon-neutrinos, which are track-like and so have a pointing better than $1^{\circ}$, are at galactic latitudes larger than $30^{\circ}$, i.e. $|b|>30^{\circ}$.

Flavor Composition Current data still allow for a variety of flavor mixing scenarios. Only a scenario of only-electron-neutrino production (present in the case of neutron decay dominance is outside of the 95\%-confidence level range of the measurement [3].

Temporal features No temporal features have been detected in the IceCube signal so far. This means that both source scenarios with steady-state emission and flaring states are possible at this point.

\section{Galactic}

Both point sources and truely diffuse fluxes contribute to the measured neutrino signal. Concerning a truely diffuse component, neutrinos from interactions of diffuse cosmic rays with the interstellar medium are expected to be the dominent source, as recently calculated in [5]. These calculations use updated hadronic cross-sections and primary composition models. The authors 
show that the flux is less than $0.1 \%$ of the detected signal. More optimistic models, using GALPROP for a more detailed gas map model in the Galaxy still predict on the order of a percent contribution from such a diffuse Galactic component [6]. All calculations agree in the sence that it is clear that this signal cannot make up the entire detected diffuse neutrino flux. Concidering all supernova remnants as individual sources to contribute as a quasi-diffusive flux, the individual spectral energy distributions can be fitted, considering synchrotron emission at radio to X-ray energies and a combination of bremsstrahlung, Inverse Compton scattering and $\pi^{0}$-decays. 24 Supernova Remnants (SNRs) could be identified with a potential dominant hadronic component. Extrapolating the results to a population of around 200 SNRs that should contribute to a diffuse neutrino flux from cosmic ray interactions directly at the SNR shock fronts results in a flux that can be slightly higher than the fully diffuse component in the Milky Way, in particular between 100 and $1000 \mathrm{GeV}$, but the contribution is still at a percentlevel with respect to the measured diffuse flux, at maximum. These examples are in line with other calculations, which all show that the Galactic component on total should be subdominent in the total neutrino spectrum.

\section{Extragalactic Sources}

There are two central and solid conclusions from the non-detection of point sources with IceCube concerning potential extragalactic sources of cosmic rays: (1) Neutrinos from the prompt emission phase of gamma-ray bursts (GRBs) can only contribute to the diffuse flux at the percentlevel or below [7]; (2) the contribution of Fermi-detected blazars to the diffuse flux can be constrained to less than $27 \%$ [8]. This does not mean that blazars are excluded in general, as the sample which is selected at $\mathrm{GeV}$ energies is likely to be dominated by leptonic emission (IC/bremsstrahlung). Active Galaxies are among the most interesting candidates for high-energy neutrino emission, in particular as they are one of the central candidate sources for the emission of ultra-high energy cosmic rays. Radio loud galaxies provide jets in which cosmic rays can be accelerated and different photon fields (disk, torus, broad/narrow line emission regions) as well as denser hydrogen targets (disk, torus, knots) provide targets for neutrinos to interact. Considering that photohadronic interactions result in a flat spectrum, i.e. much flatter than $E^{-2}$, below the break energy $E_{V, \text { break }} \approx 0.8 \mathrm{PeV} \cdot(\Gamma / 10)^{2} \cdot\left\langle E_{\gamma} / \mathrm{keV}\right\rangle^{-1} \cdot(1+z)^{-2}$, only photon fields with an average photon energy of $\left\langle E_{\gamma}>\sim \mathrm{MeV}\right.$ (or non-relativistic parts of the jet, $\Gamma \sim 1$ with $\left\langle E_{\gamma}>\sim 10 \mathrm{keV}\right.$ ) can explain the steep spectrum that is detected in IceCube up to several PeV energies. Hadronic interactions, on the other hand, have quite low optical depths in general. However, considering that charged cosmic rays diffuse in the region of interest, their path through the target can be enhanced significantly, providing effective column depths of $10^{25} \mathrm{~cm}^{-2}$. This way, Fanaroff-Riley I (FR-I) galaxies become a good candidate to produce the detected signal $[9,10]$. An indication that a significant part of the detected neutrino flux must come from so-called hidden sources comes from the fact that the neutrino flux potentially exceeds the detected diffuse gamma-flux [11]. These arguments still rely on extrapolations as the detected energy ranges are different. The interesting alternative to active Galaxies is the source class of starburst galaxies, suggested in [12]. While starbursts should be subdominant as cosmic ray sources, they provide a high-density target and might produce a large neutrino flux. In that case, neutrino sources and those cosmic ray sources as detected at Earth 
would not be identical. Future measurements will show which scenario is true - a mixture of the different models presented is certainly an option as well.

\section{Prospects}

Future neutrino telescopes, in particular KM3NeT and IceCube-Gen2, will be able to solve those questions still open at this moment. The most important goal is to identify individual neutrino sources, as this will provide us with sources that can be identified as cosmic ray accelerators in the $\mathrm{TeV}$ to $\mathrm{PeV}$ range. In the Galaxy, the search for extended source regions seems most crutial, in particular concerning star-forming regions like Cygnus [13] and the Galactic Center. For extragalactic sources, the identification of active Galaxies as neutrino emitters relies on a proper selection of the sources using multimessenger information of the energy dependence and temporal evolution of the sources, in combination with the proper theoretical modeling of the latter. Combining these pieces of evidence will lead to the identification of the first neutrino point (and/or extended) sources.

Acknowledgements Thank you to the Young Academy (Berlin, Germany) for making the trip to NOW 2016 possible. Further thanks to the funding agencies, making the physics described in this review possible: BMBF, FKZ 05A14PC1; Ruhr Astroparticle and Plasma Physics Center (RAPP Center, MERCUR St-2014-004); Research Department of Plasmas with Complex Interactions (Bochum, Germany).

\section{References}

[1] Becker J K 2008 Phys. Rep. 458173.

[2] Aartsen M G et al. (IceCube) 2013 Science 3421242856 (ArXiv:1311.5238).

[3] Aartsen M G et al. (IceCube) 2015 Astrophys. J. 80998.

[4] Aartsen M G et al. (IceCube) 2016 (ArXiv:1607.08006).

[5] Joshi J C, Winter W \& Gupta N 2014 MNRAS 4393414.

[6] Ahlers M et al. 2016 Phys. Rev. D 93013009.

[7] Aartsen M G et al. (IceCube) 2015 Astrophys. J. 805 L5 (ArXiv:1412.6510).

[8] Aartsen M G et al. (IceCube) 2016 (ArXiv:1611.03874).

[9] Becker Tjus J et al. 2014 Phys. Rev. D 8912123005.

[10] Hooper D 2016 JCAP 9002.

[11] Murase K, Guetta D \& Ahlers M 2016 Phys. Rev. Lett. 116071101 (ArXiv:1509.00805).

[12] Loeb A \& Waxman E 2006 JCAP 53.

[13] Gündüz M et al. 2017 Neutrinos and gamma-rays from the Cygnus-X complex, submitted to ApJ. 